\title{
Essays \\ Ars necandi und ars moriendi in den Samuelbüchern
}

\author{
WALTER DiETRICH*
}

The paper examines the Books of Samuel from two perspectives by posing two sets of questions: when is it legitimate (or, when is it perceived to be legitimate) to kill people, and when is this strictly forbidden? And how come to terms with one's own mortality? The 'ars necandi' refers to four distinct areas: killing in war, suicide, murder and execution. Murder is absolutely prohibited, executions are best avoided, killing in war should be limited as much as possible, whilst suicide is not evaluated. The 'ars morendi' distinguishes between premature death, which hits hard and is difficult to process, and death in old age, which is desirable, but needs to be carefully prepared for nonetheless. David himself is threatened by a thousand deaths; his survival is a miracle, his eventual death a soberly noted fact.

Keywords: Killing and dying, assassination, liquidation, suicide, warfare, premature death, old age, grief, David, Books of Samuel.

\section{Einführung}

Wohl nirgendwo in der Bibel ist der Tod so allgegenwärtig wie in den Samuelbüchern. Fast kein Kapitel, in dem nicht Menschen auf irgendeine Weise zu Tode kommen oder ihm nur ganz knapp entkommen. Offenbar wollen die Erzähler nicht nur schildern, wie die Menschen in der frühen Königszeit Israels gelebt haben, sondern auch wie sie gestorben sind. So sehen wir nicht nur die Hauptfiguren - Samuel, Saul, David - sterben, sondern genauso zahllose Nebenfiguren. Mit der Binsenweisheit, dass der Mensch sterblich ist, wird hier bedingungslos ernst gemacht. Der Grund dafür ist kaum, dass die Autoren der Samuelbücher etwa makaber morbide Neigungen hatten, sondern vielmehr, dass ihnen an einem ernsthaften, realistischen Umgang mit dem Thema Tod lag. Vermutlich wollten sie ihren Leserinnen und Lesern etwas sagen über eine angemessene Haltung gegenüber dem Sterben: gegenüber dem ,schlechten', das Leben zerstört, genauso wie gegenüber dem ,guten', das gleichsam zum Leben gehört.

Die einschlägigen Äußerungen der Samuelbücher lassen sich grob in zwei Bereiche aufteilen: den des Tötens und den des Sterbens. Das eine Mal

\footnotetext{
*Walter Dietrich, emeritierter Professor für Altes Testament an der Theologischen Fakultät der Universität Bern. Adressen: Post: Prof. Walter Dietrich, Nesslerenweg 16, CH-3084 Wabern/Schweiz; E-mail: walter.dietrich@theol.unibe.ch
} 
wird der Tod zugefügt, das andere Mal erlitten. Oft sind das natürlich zwei Seiten einer Medaille. Doch der Erzählfokus richtet sich einmal mehr auf die eine, einmal mehr auf die andere Seite. Es möge nicht als zynisch empfunden werden, dass der erste der beiden Bereiche mit „ars necandi“ überschrieben ist. Wenn man den Ausdruck nicht rein technisch missversteht (Wie bringt man am besten Menschen um?), ist er durchaus sinnvoll: Wann ist bzw. galt damals Töten als unvermeidlich und zulässig, wann als unzulässig und unbedingt zu vermeiden? Auch bei der „ars moriendi“, der Kunst des Sterbens, ist zu differenzieren: zwischen einem Tod, der zu erwarten ist und vorbereitet werden kann, und einem unerwarteten, vorzeitigen Tod, und wie dieser verarbeitet werden kann.

\section{Ars necandi in den Samuelbüchern}

\subsection{Kriegstod}

Am massenhaftesten gestorben wird in den Samuelbüchern im Krieg. Die Zeit der Staatenbildung in Israel scheint eine ungewöhnlich kriegerische Zeit gewesen zu sein. Gleich zu Beginn wird von einer verlorenen Schlacht der noch nicht von einem König geführten Stämme Israels gegen die Philister berichtet. 30.000 Mann sollen gefallen (1 Sam 4,10), zudem das gesamte führende Priestergeschlecht der Eliden ausgerottet worden sein. Doch wie ein Funken Glut unter der Todesasche erscheint die Geburt eines Eliden im Augenblick der Katastrophe (1 Sam 4,19-22).

Israel überlebt, schwer lädiert, die Krise und will alsbald einen König haben, der „vor uns ausziehen und unsere Kriege führen soll“ (1 Sam 8,20). Samuel kommt diesem Wunsch widerstrebend nach und kürt Saul zum ersten König Israels. Dessen erste Großtat ist ein Sieg über die Ammoniter, bei dem ungezählte Krieger dieses östlichen Nachbarvolkes ihr Leben verlieren und von den Überlebenden „nicht zwei beieinander blieben“ (1 Sam 11,11). Bald darauf folgt ein Krieg gegen die Philister im Westen, den Saul zwar gewinnt, bei dem er aber erste Blessuren erleidet (1 Sam 13f.), danach einer gegen die Amalekiter im Süden, die Saul angeblich mit Kind und Kegel ausrotten sollte, was er nicht ganz vollständig tat (1 Sam 15). In einem Summarium werden die Nachbarvölker noch einmal aufgezählt, gegen die Saul Krieg führte (1 Sam 14,47f.).

Der Anfang von Davids Karriere ist in Sauls Kämpfe mit den Philistern verwoben. Den Auftakt bildet die Tötung Goliats, eines ihrer Vorkämpfer, und die anschließende Verfolgung des flüchtenden Feindes, wobei „Erschlagene auf dem Weg lagen von Schaarajim bis Gat" (1 Sam 17,52). Um die Hand der Prinzessin Michal zu gewinnen, liefert David eine schauerliche 
Brautgabe in Form von 200 Philister-Vorhäuten ab (1 Sam 18,27). Diese und noch weitere Kriege mit den Philistern übersteht David unbeschadet (2 Sam 5,17-25; 21,15-22), während Saul zusammen mit dreien seiner Söhne und ungezählten Soldaten diesem Feind zum Opfer fällt (1 Sam 31). Die Ammoniter, die schon Saul einmal geschlagen hat, unterwirft David endgültig (2 Sam 12,26-31). Auch bei David fasst ein Summarium alle Kriegsgegner zusammen (2 Sam 8), zu denen jetzt auch die Aramäer im Norden zählen (vgl. auch 2 Sam 10). Besonders erschreckend sind hier die Nachrichten von Massentötungen an Moabitern (2 Sam 8,2) und Edomitern (2 Sam 8,13). In diesem Summarium noch nicht einmal aufgeführt sind die Bürgerkriege in der Zeit Davids, die noch einmal zahlreiche Opfer forderten (2 Sam 2; 18).

Von all diesen Kriegsereignissen berichten die Erzähler der Samuelbücher scheinbar ungerührt. Und sie lassen es als natürlich erscheinen, dass um tote Israeliten getrauert und über tote Feinde gejubelt wird. David beklagt die Niederlage Israels und den Tod Sauls und Jonatans (2 Sam 1,17-29), die Frauen Israels rühmen Saul und David als die, die „Tausende“ bzw. „Zehntausende“ erschlagen haben (1 Sam 18,7, vgl. 21,12; 29,5). Moralische Skrupel scheinen nicht zu bestehen. „Das Schwert frisst bald hier, bald dort“, bemerkt David selbst lakonisch (2 Sam 11,25).

An einigen Stellen aber ist doch ein Unbehagen über die Massenhaftigkeit und Wahllosigkeit des Sterbens im Krieg zu spüren. Gelegentlich wird das Bestreben sichtbar, bei einer militärischen Auseinandersetzung nur den Anführer des Feindes zu töten - in der Hoffnung, dass dann seine Soldaten die Flucht ergreifen und so ein großes Blutvergießen vermieden werden kann (2 Sam 17,1-3; 18,3; 20,20f.). Zweimal gibt es den Versuch, eine Feldschlacht zu umgehen, indem man Stellvertreter miteinander kämpfen lässt, die Sieg und Niederlage unter sich ausmachen sollen; einmal macht Goliat dem Heer Sauls diesen Vorschlag (1 Sam 17,8f.), das andere Mal der Heerführer Eschbaals, Abner, dem Heerführer Davids, Joab (2 Sam 2,14). In beiden Fällen endet der Stellvertreterkampf freilich doch in einem allgemeinen Blutvergießen. Daraufhin macht im zweiten Fall Abner seinem Gegenspieler Joab einen noch weitergehenden Vorschlag: Man solle doch das Kämpfen gänzlich einstellen (2 Sam 2,26f.) - und Joab lässt sich auch darauf ein. Hinter solchen Erzählzügen steht unverkennbar die Sehnsucht nach einer Begrenzung oder gar Beendigung des Tötens und Sterbens im Krieg.

$\mathrm{Ob}$ sich die vielen Kriege zur Frühen Königszeit hätten vermeiden lassen, diskutieren die Erzähler nicht ausdrücklich. Wohl aber geben sie zu verstehen, dass die Frage nach der Kriegsschuld nicht unwichtig ist. Den ersten großen Krieg gegen die Philister, den Israel verlor, hat es selbst vom Zaun 
gebrochen (1 Sam 4,1). Sauls und Jonatans Schlag gegen sie war die Antwort auf ihr freches Vordringen mitten in israelitisches Siedlungsgebiet (1 Sam 13,2-5.17-23). Goliat musste fallen wegen seiner Überheblichkeit (1 Sam 17,8-10.43-47). Die Niederlagen der Ammoniter gegen Saul und David waren selbstverschuldet (1 Sam 11,1f.; 2 Sam 10,1-4). Abschalom entfesselte einen Bürgerkrieg gegen seinen Vater, und Jhwh selbst sorgte dafür, dass er ihn verlor (2 Sam 17,14). Offenbar werden die Toten solcher Kriege dem Aggressor angelastet, nicht dem, der sich gegen den Angriff zur Wehr setzt.

\subsection{Selbsttötung}

Die Samuelbücher schildern zwei prominente Fälle von Suizid. Einmal nimmt sich ein König das Leben, einmal ein königlicher Ratgeber.

König Saul macht in den Erzählungen der Samuelbücher zunehmend den Eindruck eines gequälten Menschen. Jhwh habe einen „schlimmen Geist" über ihn geschickt, der ihn handlungsunfähig und unberechenbar machte (1 Sam 16,14.23; 18,10). Nach und nach wenden sich alle von ihm ab, die ihn früher unterstützt haben: Samuel, David, seine eigenen Kinder, das Volk, Gott. Vor der Entscheidungsschlacht gegen die Philister läuft er in einer düsteren Vorahnung zu einer Totenbeschwörerin, um seinen einstigen Mentor Samuel aus der Unterwelt heraufholen zu lassen; doch dieser kündigt ihm nur die bevorstehende Katastrophe an (1 Sam 28). Tatsächlich überrennen die Philister Israels Heer, Saul wird gestellt, wohl auch verwundet; als sein Waffenträger sich weigert, ihm den Gnadenstoß zu geben, stürzt er sich ins eigene Schwert (1 Sam 31,4). Die Erzählung enthält sich jeglicher Bewertung des Vorgangs, scheint vielmehr Verständnis zu zeigen für den König, der „diesen Unbeschnittenen“ nicht lebendig in die Hände fallen wollte (1 Sam 31,4). Sein Freitod, auch wenn er nicht wirklich aus freien Stücken erfolgte, hat etwas Heroisches. Eine andere Version seines Endes, die ein Amalekiter (ausgerechnet ein Amalekiter!) zum Besten gibt: dass nämlich er Saul auf dessen Bitten hin den Todesstoß versetzt habe (2 Sam 1,6-10), erscheint als unaufrichtig und als Herabwürdigung des Saul'schen Helden-Images.

Auch der andere Suizid wird ohne jeden bewertenden Unterton dargestellt. Ein hoch angesehener Berater Davids, Ahitofel, hat beim Aufstand Abschaloms dessen Partei ergriffen: ein prekärer, aber nicht von vornherein unverständlicher oder verwerflicher Schritt; offenbar standen praktisch ganz Israel und Juda auf Abschaloms Seite, wohingegen sich David faktisch nur mehr auf seine Söldnertruppen stützen konnte - und auf eine kleine Fünfte Kolonne, die er in die Umgebung des Aufrührers einschleuste. Ihr gelang es, den Einfluss Ahitofels zu dämpfen und Abschalom zu verhängnisvollen Entscheidungen zu veranlassen. Ahitofel erkannte sofort, dass damit alles verlo- 
ren war; „er sattelte seinen Esel, machte sich auf und ging zu seinem Haus, zu seiner Stadt, und traf Anordnungen über sein Haus und erhängte sich " (2 Sam 17,23). Sein Handeln erscheint als vollkommen folgerichtig, so als habe er gar nicht anders gekonnt, als seinem Leben ein Ende zu setzen. Den Leser, die Leserin überkommt ein Schauder: nicht nur über das traurige Ende dieses offenbar außergewöhnlich begabten Menschen, sondern auch über seine Einschätzung Davids, dessen Rache er sich auf keinen Fall aussetzen wollte.

\subsection{Mord}

Durch die Samuelbücher zieht sich eine Kette erschreckender Mordtaten. Die Mordopfer sind, ebenso wie die Mörder, sämtlich prominent. (Man kann sicher sein, dass es noch mehr Morde gab, nur fanden sie keinen Eingang in die Geschichtsschreibung.) Auffälligerweise waren alle drei ersten Könige in Morde verwickelt - so als ob Machthäufung fast unvermeidlich zu Machtmissbrauch führte.

Von Saul werden verschiedene Mordversuche gegen David berichtet, die freilich sämtlich fehlschlugen. David seinerseits hat den Ehemann einer von ihm geschwängerten Frau aus dem Weg geräumt; der Batscheba-Urija-Skandal ist wohl der dunkelste Fleck auf seiner Weste (2 Sam 11). Zwar versucht der König zunächst beinahe verzweifelt, das Problem auf andere Weise zu lösen: Urija soll partout zum Beischlaf mit Batscheba bewegt werden, damit ihm die Vaterschaft untergeschoben werden kann. Doch als dies nicht gelingt, greift David zu einem perfiden Mittel: Er lässt den offenbar arglosen Mann sein eigenes Todesurteil an den Heerführer Joab übermitteln, der ihn daraufhin, scheinbar unverdächtig, im Krieg fallen lässt. Doch mindestens Joab weiß Bescheid über die wahren Hintergründe - und ebenso der Prophet Natan, der den Mörder zur Rede stellt (2 Sam 12), und danach ungezählte Leserinnen und Leser.

Salomo, das zweite Kind aus der unheiligen Liaison mit Batscheba, beerbt David. Er gelangt auf reichlich undurchsichtige Weise an die Macht und lässt die beiden führenden Vertreter einer Gegenpartei, die eine andere Thronfolgeregelung anstreben, ermorden (1 Kön 2,25.34). Sein Mordarm ist in beiden Fällen der General Benaja. Überhaupt fällt auf, dass die den Königen angerechneten Morde nie von diesen selbst, sondern immer von anderen ausgeführt werden. Wer die Macht hat, muss sich nicht selbst mit Blut bespritzen. Eben dies gilt auch vom Prinzen Abschalom, als dieser sich seines älteren (Halb-)Bruders Amnon entledigen will. Amnon hat nicht nur Abschaloms Schwester, Tamar, vergewaltigt, er versperrt als Erstgeborener Davids dem ehrgeizigen Zweiten den Weg zum Thron. Die Bediensteten, die den Mord ausüben sollen, muss Abschalom nachdrücklich ermutigen: „Fürchtet euch nicht 
- habe nicht ich es euch befohlen? Habt Mut und seid stark!“ (2 Sam 13,28) - woraufhin die Leute tun, was von ihnen erwartet wird.

Demgegenüber legt der General Joab bei drei Morden, die ihm angelastet werden, selbst Hand an. Zwei Konkurrenten um das Amt des Armeechefs ermordet er meuchlings, indem er sie scheinheilig begrüßt und ihnen unversehens einen Dolch in den Bauch stößt (2 Sam 3,27; 20,9f.). Dem rebellischen Prinzen Abschalom, der sich mit seinem langen Haupthaar im Geäst eines Baumes verfangen hat und dort hilflos zwischen Himmel und Erde schwebt, stößt er drei "Stäbe“ ins Herz, die ihn noch nicht töten, doch erledigen das dann seine Waffenträger (2 Sam 18,14f.).

Besonders niederträchtig verhalten sich zwei Offiziere, die den Sohn und Nachfolger Sauls, Eschbaal, im Mittagsschlaf ermorden, ihm den Kopf abschneiden und diesen erwartungsvoll zu David bringen; doch der lässt sie unverzüglich liquidieren (2 Sam 4). Mit Mördern will David nichts zu tun haben - was ihn freilich nicht hindert, die Nachfolge des Ermordeten anzutreten (2 Sam 5,1-3).

Alle geschilderten Mordtaten werden von den biblischen Erzählern als irgendwie begründet, in gewisser Weise verständlich, nie aber als berechtigt hingestellt. Mord kann nicht legal und schon gar nicht legitim sein. Anders als Soldaten im Krieg oder Selbstmörder bewegen sich Mörder klar außerhalb der ars necandi.

\subsection{Liquidation}

Gemäß den Samuelbüchern zieht kein Mörder bleibenden Gewinn aus seiner Tat. Über kurz oder lang ereilt sie alle das Schicksal, das sie mit ihrer Bluttat selbst über sich heraufbeschworen haben. Der Mörder Saul stirbt auf dem Schlachtfeld von eigener Hand (1 Sam 31). Der Mörder David kommt glimpflicher davon; zwar spricht er sich (unwissentlich) selbst das Todesurteil, doch wird dieses, als er seine Tat bereut, auf das im Ehebruch gezeugte Kind verschoben (2 Sam 12,1-14; der Vorgang wird uns noch beschäftigen). David seinerseits lässt Mörder, die sich selbst überführt haben, sofort hinrichten (2 Sam 1,15; 4,12). An General Joab, den mehrfachen Mörder, traut er sich nicht heran, doch empfiehlt er seinem Sohn Salomo dessen Liquidierung (1 Kön 1,5f.). Der Brudermörder Abschalom wird durch selbstgewähltes Exil und lang anhaltenden väterlichen Liebesentzug bestraft (2 Sam 13,37-39; 14,32f.) - die Erzähler lassen offen, ob diese Maßnahmen streng genug waren; am Ende jedenfalls stirbt auch Abschalom einen elenden Tod.

In einem einzigen Fall erscheint eine Tötung als einigermaßen legitim, obwohl der Betreffende sich keines Mordes, sondern ,nur' des Aufruhrs schuldig gemacht hat: Der Benjaminit Scheba ben Bichri ruft nach den Wirren 
des Abschalom-Aufstands die Nordstämme Israels zur Sezession auf (2 Sam 20,1f.). David ordnet an, ihn sofort unschädlich zu machen - eine Aufgabe, die ungefragt Joab übernimmt. Als Scheba sich in eine Stadt zurückzieht und Joab diese zu erstürmen sich anschickt, werfen ihm die Bewohner den Kopf des Rebellen über die Mauer zu (2 Sam 20,22).

Fast stärker noch als vollstreckte Hinrichtungen stechen Fälle ins Auge, in denen auf Liquidationen verzichtet wird. Nachdem Saul zum König gekürt worden ist, verweigern ihm Personen, die mit dem wenig schmeichelhaften Titel $b^{e} n \hat{e} b^{e}$ lijja'al (soviel wie „Aufrührer, Terroristen“) belegt werden, die Huldigung (1 Sam 10,27). Nach seinem triumphalen Sieg über die Ammoniter wollen seine begeisterten Anhänger an diesen Dissidenten ein Exempel statuieren; doch Saul verbietet ihre Tötung mit dem Hinweis auf Jhwh, der den Sieg geschenkt habe (1 Sam 11,12f.). Diese Begründung ist bemerkenswert: Ein Machthaber, der sich Gott unterstellt fühlt, tötet weniger leicht.

Später allerdings muss Saul von anderen am Töten gehindert werden. Seinen Sohn Jonatan, der einen Sieg über die Philister herbeigeführt, dabei aber eigensinnig gehandelt und gar noch ein königliches Gebot übertreten hat, verurteilt er zum Tode; doch das Kriegsvolk verhindert die Hinrichtung (1Sam 14,44f.). Auch über die Priester von Nob fällt Saul ein Todesurteil - subjektiv einigermaßen nachvollziehbar (er kann von ihrer Konspiration mit David überzeugt gewesen sein), objektiv aber ein Fehlurteil und ein Sakrileg. Zwar weigern sich seine "Läufer“ (die Leibwache), die Strafe zu vollstrecken, doch dann findet sich doch ein williger Exekutor: der Edomiter Doëg. Seinen Widersacher David sucht Saul gleich selbst zu töten (1Sam 19,9f.); doch da fallen ihm seine Kinder, Michal (1Sam 19,11-17) und Jonatan (1Sam 20) in den Arm.

David gelingt es, sich vor dem ihn wütend verfolgenden Saul ins judäische Bergland zurückzuziehen. Dort ergeben sich wiederholt Situationen, in denen der Jäger zum Gejagten wird. Zweimal könnte David Saul problemlos umbringen, wird dazu sogar ausdrücklich aufgefordert, weigert sich aber - bezeichnenderweise wieder unter Verweis auf Jhwh, dessen „Gesalbter“ Saul sei (1 Sam 24,7; 26,9). Zwischen die beiden Erzählungen von Sauls Verschonung schiebt sich eine dritte ähnlichen Inhalts: Ein reicher Viehzüchter namens Nabal hat David schwer provoziert; als dieser mit seiner Truppe anrückt, um blutig Rache zu nehmen, tritt ihm Nabals Gattin Abigajil in den Weg, besänftigt ihn und hält ihn mit kluger Rede davon ab, „in Blutschuld zu geraten“ und sich „mit eigener Hand zu helfen“ (1 Sam 25,26.33). Diese Frau lehrt David eine wichtige Lektion: dass ein großer Herrscher nur sein kann, wer sich selbst beherrschen kann. 
Später zeigt David (der biblische David, wohlgemerkt!) verschiedene Male, dass er die Lektion gelernt hat. Gelegenheit dazu gibt ihm insbesondere der Aufstand Abschaloms, der zunächst enorm erfolgreich verläuft, am Ende aber scheitert. Zu Beginn, als David scheinbar geschlagen das Feld räumt, zeigen ihm einige Personen unverhohlen ihre Antipathie (2 Sam 16,1-12); als er siegreich zurückkehrt, rächt er sich nicht, sondern lässt sie am Leben (2 Sam 19,17-31).

\section{Ars moriendi in den Samuelbüchern}

\subsection{Vorzeitiger Tod}

Viele Tode in den Samuelbüchern kommen eindeutig zu früh. Das gilt natürlich von allen Mordopfern, aber auch von den Vielen, namentlich Genannten wie Namenlosen, die ihr Leben im Krieg verlieren. Hinzu kommen einzelne krankheitsbedingte Todesfälle oder der Tod im Kindsbett. Die Samuelbücher schildern diese traurigen Ereignisse, berichten aber auch, wie sie verarbeitet und überwunden wurden.

Die hochschwangere Ehefrau des elidischen Priesters Pinhas bricht bei der Nachricht von dessen Tod zusammen; es überkommen sie die Wehen, und im Sterben gebiert sie einen Sohn. Die Nachricht davon kann sie nicht mehr trösten; nur einen trostlosen Namen (Ikabod, „Wo ist die Herrlichkeit?") vermag sie dem Kind noch zu geben (1 Sam 4,19-21). Außer diesem Eliden überlebt auch der junge Samuel die katastrophale Niederlage gegen die Philister; er ist im Haus Elis aufgewachsen und bereits zum geistlichen Führer Israels berufen (1 Sam 3). Die Todesstunde der Frau des Pinhas und Zehntausender Israeliten wird so zur Geburtsstunde eines neuen Israel.

Samuel setzt den ersten König ein. Dieser erlebt einen steilen Aufstieg, dann aber bald einen unaufhaltsamen Abstieg. Am Ende verliert nicht nur er sein Leben, sondern praktisch seine gesamte Familie. Diese Tragödie macht den Weg für David frei, der aber darüber, glaubt man der biblischen Darstellung, nicht etwa glücklich, sondern tieftraurig ist. Diejenigen, die Morde an Mitgliedern der Königsfamilie zu verantworten hatten, lässt er liquidieren (2 Sam 1,15; 4,11). Joab, der Abner, Sauls Onkel und Heerführer ermordet hatte, zwingt er zur Teilnahme an einer von ihm verordneten Staatstrauer (2 Sam 3,31). Bei dieser stimmt er ein Trauerlied an, in dem er öffentlich beklagt, dass Abner gefallen sei „wie vor Bösewichtern“ (2 Sam 3,34) - eine deutliche Distanzierung von Joab und seiner Bluttat. (Mehr wollte oder konnte David gegen seinen ebenso treuen wie rabiaten Gefolgsmann nicht unternehmen.)

Noch weniger als gegen Joab vermochte David gegen die Philister auszurichten, die Saul und Jonatan auf dem Gewissen hatten. In seinem Trau- 
erlied auf diese beiden fordert er symbolisch dazu auf, die Siegesnachricht keinesfalls den Philisterstädten zu überbringen, damit die Frauen dort keinen Grund zur Freude hätten (2 Sam 1,20). Im Übrigen rühmt er die Gefallenen als einander zugetane, überaus tapfere und um Israel hochverdiente Helden (2 Sam 1,22-24). Insbesondere Jonatans Tod erfüllt ihn mit tiefem Schmerz, den er nicht allein tragen, sondern mit allen teilen will, die ihm zuhören. In aller Offenheit und Missverständlichkeit bekennt er, dass ihm Jonatans Liebe kostbarer gewesen sei als Frauenliebe (2 Sam 1,26).

In geradezu heldenhafter Art leistet Rizpa, eine Nebenfrau Sauls, Trauerarbeit. Sie muss erleben, dass ihre beiden Söhne zusammen mit fünf weiteren Sauliden wegen einer angeblichen Blutschuld des Hauses Saul hingerichtet werden. Die Leichname sollen den wilden Tieren überlassen werden: eine weitere schlimme und demütigende Strafe, galt doch eine ordentliche Bestattung als Voraussetzung für den Eintritt ins Totenreich. Rizpa lässt sich - vermutlich gegen ausdrückliches Verbot - an der Hinrichtungsstätte nieder und weicht nicht, bis David sich endlich zur Beisetzung der Leichen entschließt (2 Sam 21,1-14).

Geradezu ein Lehrbeispiel für den Umgang mit Sterben und Tod ist die Erzählung von David und seinem ersten Kind mit Batscheba. Natan hatte den Tod dieser Frucht einer verbotenen Beziehung angekündigt (2 Sam 12,14), und prompt „schlug Jhwh das Kind, das die Frau Urijas (!) David geboren hatte" (2 Sam 12,15), d.h. das Neugeborene wird krank. David, der den Grund genau kennt, ist nicht bereit, diese Strafe für seine Schuld zu akzeptieren - und der moderne Mensch pflichtet ihm bei: Was kann ein Kind für das, was seine Eltern getan haben? Die Bibel kennt diese Frage durchaus (vgl. Ez 18), doch in diesem Fall argumentiert bzw. erzählt sie (noch) ganz archaisch. David ringt mit aller Kraft um das Überleben des Kindes: Er „sucht Gott", fastet, schläft auf dem Fußboden und lässt sich von alledem durch niemanden abbringen (2 Sam 12,16f.; die Wissenschaft spricht hier von „Selbstminderungsriten", die darauf abzielen, das Mitleid der Gottheit zu wecken). Bis zu diesem Punkt ist die Erzählung, existenziell gedeutet, eine Aufforderung, sich dem vorzeitigen Tod eines Menschen, zumal eines Kindes, mit allen Mitteln (heute würde man wohl medizinische Mittel den geistlich-rituellen vorziehen) entgegenzustemmen. Doch im Fall des Erstgeborenen Batschebas (wie auch in vielen anderen Fällen) bleibt Gott unerbittlich (bzw. ist die Medizin machtlos): „Am siebten Tag starb das Kind“ (2 Sam 12,18). Die Diener wagen das David gar nicht zu sagen, weil sie einen hoch emotionalen, womöglich (selbst)zerstörerischen Ausbruch von Trauer befürchten. Doch der König reagiert ganz unerwartet: Er wäscht und salbt sich, geht ins 
Haus Jhwhs, um anzubeten (nicht mehr: um mit Gott zu ringen!) und lässt sich Speisen und Getränke bringen (2 Sam 12,20). Auf verblüffte Nachfragen erklärt er: „Als das Kind noch lebte, fastete und weinte ich, weil ich mir sagte: Wer weiß, vielleicht merkt es Jhwh und erbarmt sich meiner, und das Kind bleibt am Leben. Jetzt aber, wo es tot ist: Was soll ich fasten? Kann ich es wieder zurückholen? Ich, ich gehe wohl zu ihm, es aber, es kehrt nicht zu mir zurück" (2 Sam 12,22f.). Das ist eine bemerkenswerte Ergebung in die Unwiderruflichkeit des Todes, gemildert durch keine noch so leise Hoffnung auf Totenauferstehung. Und diese Nüchternheit führt zu einer erstaunlichen Fähigkeit, durch schweres Leid hindurch ins Leben zurückzufinden. David denkt sogar an die schmerzerfüllte Mutter und „tröstet“" sie, indem er zu ihr „eingeht“, woraufhin sie prompt schwanger wird (2 Sam 12,24). Wohl mag man fragen, ob hier nicht ein Mann ,typisch männlich' agiert und die psychische Lage einer Frau verkennt, der ihr Kind genommen wurde; immerhin aber wird das zweite Kind Batschebas Salomo, der spätere Thronerbe.

Im Verlauf der Zeit werden noch zwei weitere Söhne Davids sterben: nicht mehr als Kleinkinder, sondern als junge Erwachsene - und gleichwohl viel zu früh. Der eine, sein Ältester Amnon, ein Vergewaltiger, wird bei einem Fest umgebracht, zu dem alle Königssöhne eingeladen waren. Daraufhin gelangt nach Jerusalem das Gerücht, sie seien alle tot. David zerreißt sein Gewand und wirft sich zur Erde. Dann stellt sich heraus, dass ,nur' Amnon tot ist, doch genügt dies, um „den König und alle seine Bediensteten in ein sehr großes Weinen“ ausbrechen zu lassen (2 Sam 13,36). Doch ändern lässt sich an dem schockierenden Faktum nichts mehr.

Der nächste Davidsohn, der stirbt, ist Amnons Mörder, Abschalom. Von seinem schrecklichen Ende, im Geäst eines Baumes hängend und von Joab und seinen Leuten erschlagen, war schon die Rede. Fast noch mehr Gewicht als auf dieses Geschehen legen die Erzähler darauf, wie David davon Kenntnis erhielt und wie er reagierte. Der König hatte nämlich ausdrücklich angeordnet, das Leben des ,jungen Mannes“ zu schonen (2 Sam 18,5). Joab hatte sich darüber hinweggesetzt und war nun in der Verlegenheit, seinen Herrn über den Gang der Dinge zu informieren. Einen Vertrauten, der sich erbot, die vermeintlich frohe Botschaft vom Sieg zu überbringen, hielt Joab zurück, weil er Schlimmes befürchtete; stattdessen schickte er einen dunkelhäutigen Söldner los. Doch es rannten beide, kamen nacheinander beim König an, und dieser wollte von ihnen nur eines wissen: wie es dem ,jungen Mann“ gehe (2 Sam 18,29.32). Als er erfasst hat, was geschehen ist, „durchfuhr es ihn, und er stieg in das Obergeschoss des Tores hinauf und weinte. Und weinend rief er: ,Mein Sohn Abschalom, mein Sohn, mein Sohn Ab- 
schalom! Ach, wäre ich doch an deiner Stelle tot, Abschalom, mein Sohn, mein Sohn!'“ (2 Sam 19,1) Diese erschütternde Klage zeigt einen hilf- und trostlosen Vater, dem sein Kind geraubt wurde. David vergisst völlig, was um ihn her vorgeht, und erst, als Joab ihn grob ermahnt, die für ihn siegreichen Soldaten nicht vor den Kopf zu stoßen, besinnt er sich auf seine Amtspflichten. Die Erzähler sagen es nicht, doch man kann es vermuten, dass er in der Folge ein gebrochener Mann war.

\subsection{Tod im Alter}

Neben den tragischen und aufwühlenden Fällen vorzeitigen Sterbens gibt es in den Samuelbüchern auch solche, in denen der Tod nicht überraschend kommt und deswegen vorab bedacht und hernach gelassener hingenommen werden kann.

Die beiden letzten Anführer Israels in der vorköniglichen Ära sterben in hohem Alter. Von Eli erfährt man sogar, welches sein Sterbejahr war: das neunundneunzigste (1 Sam 4,15). Und selbst dann wäre er noch nicht gestorben, hätte er nicht die schreckliche Nachricht von der Niederlage Israels, dem Tod seiner Söhne und vor allem vom Verlust der heiligen Lade vernehmen müssen; auf sie hin fällt er rücklings vom Stuhl und bricht sich das Genick (1 Sam 4,18). Das ist zwar immer noch tragisch, doch laut getrauert wird nicht um den toten Eli, sondern um die zu Tode gekommene Jungmannschaft Israels (1 Sam 4,13f.). Beim Ableben Samuels, das wohl ebenfalls in hohem Alter erfolgte, soll sich immerhin „ganz Israel versammelt und die Totenklage gehalten" haben (1 Sam 25,1; 28,3). Mit dem normalen Trauer- und Bestattungsritual hat es indes sein Bewenden.

Am Ende der Samuel-Erzählungen sieht man einen alten Mann sehr umsichtig mit seinem absehbaren Sterben umgehen. Barsillai, ein Notabler aus der Ortschaft Rogelim im ostjordanischen Gilead (vgl. 2 Sam 17,27), hatte David bei dessen taktischem Rückzug vor Abschalom sehr geholfen: Dank ihm und zwei weiteren wohlhabenden und einflussreichen Männern konnte der aus Jerusalem vertriebene König sich mit den ihm verbliebenen Truppen im Ostjordanland konsolidieren und von dort aus die Niederschlagung der Rebellion und die Rückkehr an die Macht in Angriff nehmen. Nach dem Sieg und vor der Rückkehr nach Jerusalem will David sich Barsillai erkenntlich zeigen und bietet ihm an, mit ihm zu kommen und sich fortan an der königlichen Tafel versorgen zu lassen. Barsillai lehnt das mit wohlüberlegten und wohlgesetzten Worten ab: „Wie viele Jahre werde ich noch leben, dass ich mit dem König nach Jerusalem hinaufzöge? Ich bin jetzt achtzig Jahre alt - werde ich da zwischen gut und schlecht unterscheiden können, so dass dein Diener schmeckt, was er isst und was ich trinke, 
dass ich noch höre die Stimme der Sänger und Sängerinnen? Und warum sollte dein Diener noch zur Last werden für meinen Herrn, den König? ... Möge dein Diener umkehren dürfen, und ich will in meiner Stadt sterben (und beigesetzt werden) im Grab meines Vaters und meiner Mutter" (2 Sam 19,36.38). So ehrenvoll und verlockend das königliche Angebot ist, der achtzigjährige Barsillai möchte nicht noch einmal ein neues Leben beginnen. Realistisch sieht er altersbedingte Einschränkungen und Beeinträchtigungen voraus. Er denkt, es sei für ihn besser, die ihm noch verbleibende, nicht mehr allzu lange Lebensspanne in der gewohnten Umgebung zuzubringen. Das Grab seiner Eltern, das auch seines sein wird, ist ein Fixpunkt, von dem er sich nicht mehr weit wegbewegen möchte. Einer, der so spricht, zeigt ein sicheres Gespür für sein Alter und eine große Gelassenheit gegenüber seinem Tod. Auf der anderen Seite möchte Barsillai den König in seiner Großzügigkeit nicht brüskieren; auch weiß er, dass nach seinem Sterben das Leben weitergehen wird. So empfiehlt er David, an seiner Stelle einen gewissen Kimham (über den man sonst nichts Sicheres weiß) mit nach Jerusalem zu nehmen und sich um ihn zu kümmern; der König willigt sofort ein (2 Sam 19,38f.).

\subsection{David, der Unsterblich-Sterbliche}

David ist unbestritten die Hauptfigur der Samuelbücher. Entsprechend können an ihm alle wesentlichen Facetten des Themas Tod entfaltet werden. Davon, wie er Menschen zu Tode brachte oder darauf bewusst verzichtete und wie er auf den Tod anderer reagierte, war im Vorangehenden schon die Rede. Jetzt soll es noch um sein eigenes Sterben (bzw. sein Nicht-Sterben und am Ende dann doch Sterben) gehen.

Laut den Samuelbüchern hat David dem Tod schier ungezählte Male ins Auge gesehen. Das beginnt mit seinem Zweikampf gegen Goliat, in dem er jedem vernünftigen Menschen als sicherer Todeskandidat erscheinen musste. Ein Leitwort in 1 Sam 17 ist das Verb „erschlagen“. Goliat fordert eingangs einen Zweikampfgegner, den entweder er „erschlägt“ oder dieser ihn (V. 9) - eine in seinen Augen natürlich nur scheinbare Alternative. Saul setzt einen Preis auf den aus, der diesen Philister „erschlägt“ (V. 25.26.27). David, der sich zum Kampf meldet und den Saul zurückzuhalten versucht, erklärt dreimal, er habe als Hirte große Raubtiere „erschlagen“ (V. 35.36) - die Situation der Lebensgefahr war ihm also von Jugend auf bekannt. Goliat kündigt ihm, als er seiner gewahr wird, an, er werde sein „Fleisch den Vögeln des Himmels und den Tieren des Feldes"vorwerfen (V. 44). David antwortet, er wolle umgekehrt ihn „erschlagen“ (V. 46) - und das tut er dann wirklich (V. 49.50.57). 
Gegen die Philister stand Davids Leben noch oft auf dem Spiel. Wie sonst hätte er „Zehntausende schlagen“ oder 200 Vorhäute von ihnen erbeuten können? In einigen Fällen saß er schon bei ihnen in der Falle, entkam aber mit List und Tücke oder durch den tapferen Einsatz seiner Getreuen (1 Sam 21,11-16; 2Sam 21,15-17). Fast noch gefährlicher für ihn als der äußere Feind war indes der innere, vor allem König Saul: sei es, dass dieser den Rivalen durch die Hand der Philister zu beseitigen versuchte (1 Sam 18,17.21), sei es, dass seine Häscher ihn unschädlich machen sollten (1 Sam 19,1.11.21), sei es, dass er ihn persönlich an die Wand zu spießen drohte (1 Sam 18,11; 19,10), sei es, dass er ihn unerbittlich durch die Wüste jagte (1 Sam 23,8.25; 24,3; 26,2). Es wirkt wie ein Wunder, dass David all diese Anschläge lebend überstand.

Als David, mittlerweile Philistervasall geworden, mit seiner Privatmiliz im Aufgebot seines Lehnsherrn gegen Israel gezogen, dann aber vorzeitig zurückgeschickt worden war, fand er die ihm zum Lehen gegebene Stadt Ziklag zerstört und menschenleer vor; eine Bande nomadischer Amalekiter hatte die Abwesenheit der Krieger zu einem Überfall genutzt, die Stadt geplündert und gebrandschatzt, Frauen und Kinder in die Wüste verschleppt. „Da erhoben David und das Kriegsvolk, das bei ihm war, ihre Stimme, und sie weinten, bis in ihnen keine Kraft mehr war zum Weinen... Und David geriet in große Bedrängnis, weil das Kriegsvolk davon sprach, ihn zu steinigen“ (1 Sam 30,4.6). Es wäre dies nicht der einzige Fall einer Meuterei gegen einen erfolglosen Anführer gewesen. Was David rettet, ist der Einfall, Jhwh zu befragen, ob und wie man die Räuber verfolgen könne; so richtet sich die Wut auf den Feind statt auf ihn (1 Sam 30,7f.). Glücklicherweise, so muss man sagen, hat die Verfolgungsjagd Erfolg.

In schwerste Lebensgefahr gerät David im Zuge des Abschalomaufstands. Er ist sich dessen bewusst, dass sein „eigener Sohn ihm nach dem Leben trachtet" (2 Sam 16,11). Ahitofel, der zu Abschalom übergelaufene Ratgeber, benennt ganz klar das Ziel seines Schlachtplans: „Ich will über ihn kommen, solange er müde und erschlafft ist, und ihn aufschrecken; und das ganze Kriegsvolk, das bei ihm ist, wird fliehen, und ich werde den König ganz allein erschlagen" (2 Sam 17,2). So wäre es wohl gekommen, hätte Ahitofel sich durchgesetzt. Doch es kam anders. Wenn dann am Ende David um den toten Abschalom mit den Worten klagt: „Wäre ich doch an deiner Stelle tot!“ (2 Sam 19,1), dann weiß jede Leserin, jeder Leser, dass dazu nur ganz wenig gefehlt hätte.

Unsterblich ist freilich auch ein David nicht. „Und König David war alt und hochbetagt, und man hüllte ihn in Decken, doch es wurde ihm nicht 
warm“ (1 Kön 1,1). In großer Offenheit berichten die biblischen Erzähler von des großen Königs Altersschwäche - und wie um ihn herum sich Parteien herausbilden, die um seine Nachfolge buhlen. Die eine Seite wird angeführt von Adonija, dem nach dem Ableben Amnons und Abschaloms ältesten Davidsohn, die andere von Batscheba und Salomo. Der alte David wirkt unbeteiligt an diesem Ringen und einigermaßen hilflos. Doch dann, zum kritischsten Zeitpunkt, kehrt die alte Kraft zurück. David ordnet die Krönung Salomos an und erteilt diesem, als er inthronisiert ist, Ratschläge bzw. Anweisungen, wie er mit verschiedenen prominenten Persönlichkeiten verfahren soll: Die einen sind zu töten, die anderen zu schonen (1 Kön 2,5-9). Dann „legte David sich zu seinen Vätern und wurde begraben in der Davidstadt... Und Salomo saß auf dem Thron Davids, seines Vaters, und sein Königtum festigte sich sehr“ (1 Kön 2,10.12).

Ende gut, alles gut, möchte man sagen. Doch lassen die Erzähler durchaus den Eindruck aufkommen, David habe dieses Ende nicht frühzeitig und nicht energisch genug vorbereitet. Es kam eher über ihn, als dass er es geplant hätte. Anscheinend konnte er sich allzu lange in der Nachfolgefrage nicht entscheiden, vielleicht auch: sich allzu schwer von der eigenen Macht trennen. Freilich, sein Erst- und sein Zweitgeborener, die natürlichen Thronprätendenten, waren vorzeitig ausgeschieden. Zumindest im Zweiten, Abschalom, hatte er selbst wohl lange seinen Nachfolger gesehen. Als dieser aber nicht warten und die Macht mit Gewalt an sich reißen wollte, da wehrte David sich entschieden. Angeblich wollte er Abschaloms Tod nicht, doch lag dieser in der Logik des Krieges, dem David nicht auswich. Der Nächste dann, Adonija, scheint eine schwächere Kopie Abschaloms gewesen zu sein; David mochte sich für ihn nicht entscheiden, trat aber auch seinen Thronambitionen nicht entgegen (1 Kön 1,5f.). Vielleicht muss man die holpernd verlaufene und fast zu spät entschiedene Thronfolge als Davids persönliche Tragik bezeichnen. Schließlich aber, so scheinen es die biblischen Erzähler zu sehen, gilt eben doch: Ende gut, alles gut.

\section{Schluss}

Im Vorstehenden wurden die Aussagen der Samuelbücher zum Thema Tod zusammen- und nebeneinandergestellt, als wären sie ein einheitliches literarisches Werk, in dem alles planvoll aufeinander abgestimmt ist. In der Tat zeigen die Samuelbücher, verglichen etwa mit den vorangehenden Büchern Josua und Richter oder den nachfolgenden Königsbüchern, eine auffallend intensive Durchdringung und dichte Verflechtung der verschiedenen Stoffe und Gestalten, und das in einer herausragend hohen literarischen Qualität. 
Trotzdem sind sie nicht von einem einzelnen Autor, gar einem Zeitzeugen, geschaffen worden, sondern sind, wie die meisten Schriften des Alten Testaments, Traditionsliteratur, d.h. in ihnen wird älteres Traditionsgut über die Zeiten hinweg immer wieder aufgenommen und neu bearbeitet. So gibt es auch zum hier verhandelten Thema in den Samuelbüchern nicht nur eine Meinung, sondern mehrere, wohl sogar viele. Umso erstaunlicher ist es, dass sich bei der Durchmusterung der einschlägigen Texte doch ein recht kohärentes Bild einstellt. Offenbar stammen sie doch weitestgehend aus einer relativ geschlossenen Erfahrungswelt. Und wahrscheinlich haben eben doch einzelne Autoren dem Ganzen sehr deutlich ihren persönlichen Stempel aufgeprägt.

Infolgedessen finden sich in den Samuelbüchern zum Thema Tod einige durchgehende Aussagelinien, die nun noch einmal hervorgehoben werden sollen. Immer wieder wird das Axiom sichtbar, dass Mord unter keinen Umständen erlaubt ist. Dies entspricht exakt dem fünften Gebot des Dekalogs, das wörtlich lautet: „Du wirst nicht morden“ (Ex 20,13; Dtn 5,17). Das ist ein sog. apodiktisches Verbot, d.h. es gilt derart unbedingt, dass es keiner Begründung und keiner Strafandrohung bedarf. Mord ist schlicht ausgeschlossen. Wer dennoch mordet, schließt sich aus der Gemeinschaft mit Gott und den Menschen aus.

Mehrfach wird Mord mit dem Tod bestraft, und nichts deutet darauf, dass sich die Erzähler von dieser Praxis distanzieren. Sie stellen die Todesstrafe, zumindest als Möglichkeit, nicht in Frage. An dieser Stelle zeigt sich eine Zeitgebundenheit, die überwunden werden kann - und auch sollte. Moderne Staaten mit ihren entwickelten Rechts- und Strafvollzugssystemen haben andere Möglichkeiten, mit schweren Straftätern umzugehen, als sie umzubringen.

Zeitgebunden kann man auch die Haltung zum Thema Krieg finden. Die Samuelbücher sind zu erheblichen Teilen Kriegsberichterstattung. Die Erzähler freuen sich an Israels Siegen und leiden unter seinen Niederlagen. Fanden sie das massenhafte Sterben im Krieg nicht grundsätzlich problematisch? Wohl doch. Die Furchtbarkeit des Krieges, das Grauen von Verwundung, Verstümmelung und Massakrierung werden so ungeschönt ins Bild gerückt, dass jedenfalls eine schwülstige Kriegsromantik nicht aufkommen kann. Auch die stille Warnung davor, Kriege leichtfertig und einseitig vom Zaun zu brechen, sollte nicht überhört werden. Und die Schilderung verschiedener Versuche, das Blutvergießen zu vermeiden oder zu begrenzen oder zu beenden, lässt durchaus Nachdenklichkeit in dieser Frage erkennen.

Am deutlichsten friedfertig zeigen sich die Samuelbücher dort, wo sie vom Verzicht aufs Töten handeln. Die „ars necandi“ erreicht ihre höchste Stufe in ihrer Nicht-Ausübung! Namentlich David hat in diesem Punkt Vorbild- 
charakter. Wohl ein dutzend Mal soll er Menschen, die ihm im Weg standen und ihm obendrein noch feindselig gesinnt waren, verschont haben. Neuere Forschungsarbeiten bezweifeln, dass er wirklich so großmütig war; die betreffenden Erzählungen seien bloße Propaganda. Dagegen kann man (literar-) historisch argumentieren: Vermutlich ist gerade die Textschicht, die am klarsten jenes Ideal vertritt, weit nach-davidisch und insofern für Propagandazwecke kaum geeignet. Zudem ist es unwahrscheinlich, dass ein Herrscher sich so lang an der Macht hält und der Nachwelt so eindrücklich in Erinnerung bleibt, wenn er nicht irgendwie doch ungewöhnlich war. Selbst wenn David aber, wie manche wollen, ein mediokrer Machthaber und skrupelloser Serienmörder gewesen wäre: Was wäre der Gewinn, das zu wissen? Ist es nicht viel gewinnbringender, von den biblischen Autoren zu lernen, was sie an der David-Gestalt aufzeigen wollen: dass Nicht-Töten besser ist als Töten?

Was die ,ars moriendi“ angeht, ist David ebenfalls Vorbild, aber auch warnendes Beispiel. Zunächst ist es förmlich eine Kunst, so viele Male dem sicheren Tod zu entgehen. Ein ,gutes' Sterben, so lehrt dies, kann erst am Ende eines erfüllten Lebens stehen. Irgendwann aber sind die Lebenskräfte auch eines David erschöpft; er kann die Zügel nicht mehr halten, muss sie in andere Hände legen. Man könnte sich vorstellen, dies hätte ihm besser gelingen können, wenn er sich vom Leben und von der Lust, es zu gestalten, etwas weniger hätte in Beschlag nehmen lassen. Andere Figuren - der Prophet Samuel, der Gileaditer Barsillai und sogar der unglücklich endende Ahitofel scheinen darin mehr Weitsicht und Geschick zu zeigen.

Der Tod ist allgegenwärtig in den Samuelbüchern; er ist die Kehrseite des Lebens. Einmal reißt er in dieses tiefe Lücken, einmal schließt er es sanft ab. Bald kommt er unerwartet vorzeitig, dann erwartbar im hohen Alter. Bei manchen klopft er viele Male an, bei manchen nur einmal, ehe er eintritt. Bei den einen denkt man, er hätte sie früher, bei anderen, er hätte sie später treffen sollen, doch wer vermag das nachzurechnen? Jedenfalls ist auf fast jeder Seite der Samuelbücher, die so prall voll sind von Leben, ein leises oder lautes memento mori zu vernehmen.

\section{Weiterführende Literatur}

\section{Kommentare:}

Shimon Bar-Efrat, Das Erste Buch Samuel, 2007 (BWANT 176); Das Zweite Buch Samuel, 2009 (BWANT 181).

Walter Dietrich, Samuel. 1Sam 1-12, 2011 (BKAT VIII/1); 1Sam 13-20, 2012ff. (BKAT VIII/2, Lieferungen 1-7). 
P. Kyle McCarter, I Samuel, 1980; II Samuel, 1984 (AncB).

Hans Joachim Stoebe, Das erste Buch Samuelis, 1973; Das zweite Buch Samuelis, 1994 (KAT 13.1/2).

Fritz Stolz, Das erste und zweite Buch Samuel, 1981 (ZBK.AT 9).

Monographien:

Sophia K. Bietenhard, Des Königs General. Die Heerführertraditionen in der vorstaatlichen und frühen staatlichen Zeit und die Joabgestalt in 2 Sam 2-20; 1 Kön 1-2, 1998 (OBO 163).

Walter Dietrich, Die frühe Königszeit in Israel. 10. Jahrhundert v.Chr., Stuttgart 1997 (Biblische Enzyklopädie 3).

Walter Dietrich, David. Der Herrscher mit der Harfe, Leipzig 2006 (Biblische Gestalten 14).

Walter Dietrich / Moisés Mayordomo, Gewalt und Gewaltüberwindung in der Bibel, Zürich 2005.

Pia Eckstein, König David. Eine strukturelle Analyse des Textes aus der Hebräischen Bibel und seine Wiederaufnahme im Roman des 20. Jahrhunderts, Bielefeld 2000.

Israel Finkelstein / Neil A. Silberman, David und Salomo. Archäologen entschlüsseln einen Mythos, München 2006.

David M. Gunn, King David. Genre and Interpretation, 1978 (JSOT.S 6).

David M. Gunn, The Fate of King Saul. An Interpretation of a Biblical Story, 1980 (JSOT.S 14).

Baruch Halpern, David's Secret Demons. Messiah, Murderer, Traitor, King, Grand Rapids MI / Cambridge 2001.

Sara Kipfer, Der bedrohte David. Eine exegetische und rezeptionsgeschichtliche Studie zu 1Sam 16-1Kön 2, Diss. theol. Bern 2013.

Steven L. McKenzie, King David. A Biography, Oxford 2000.

Stefan Ark Nitsche, David gegen Goliath. Die Geschichte der Geschichten einer Geschichte. Zur fächerübergreifenden Rezeption einer biblischen Story, Münster 1997.

Ilse Müllner, Gewalt im Hause Davids. Die Erzählung von Tamar und Amnon (2 Sam 13,1-22), 1997 (HBS 13).

Robert Polzin, Samuel and the Deuteronomist. A Literary Study of the Deuteronomic History, 1 Samuel, Bloomington, IN 1989. 
Robert Polzin, David and the Deuteronomist. A Literary Study of the Deuteronomic History, 2 Samuel, Bloomington, IN 1993.

Hans Andreas Tanner, Amalek. Der Feind Israels und der Feind Jahwes. Eine Studie zu den Amalektexten im Alten Testament, Zürich 2005.

Jacob L. Wright, David and His Reign Revisited, Cambridge 2013.

Sammelbände:

A. Graeme Auld / Erik Eynikel (eds.), For and Against David. Story and History in the Books of Samuel, 2010 (BETL 232).

Walter Dietrich / Hubert Herkommer (Hg.), König David-biblische Schlüsselfigur und europäische Leitgestalt, Fribourg/Stuttgart 2003.

Walter Dietrich (Hg.), David und Saul im Widerstreit - Diachronie und Synchronie im Wettstreit. Beiträge zur Auslegung des ersten Samuelbuchs, 2004 (OBO 206).

Walter Dietrich (Hg.), Seitenblicke. Literarische und historische Studien zu Nebenfiguren im zweiten Samuelbuch, 2011 (OBO 249).

Carl S. Ehrlich / Marsha C. White (eds.), Saul in Story and Tradition, 2005 (FAT 47).

Tod Linafelt / Claudia V. Camp / Timothy Beal (eds.), The Fate of King David. The Past and Present of a Biblical Icon, 2010 (LHBOT 500). 